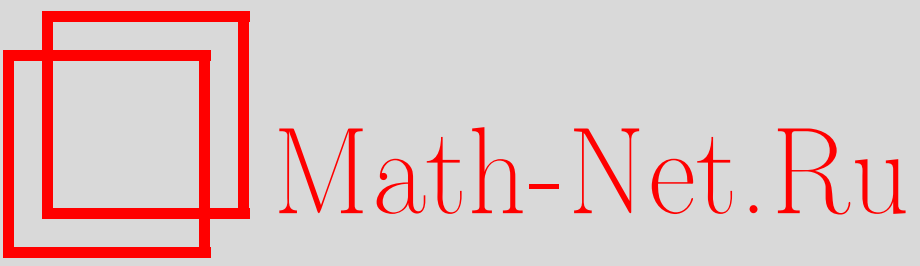

С. А. Зайцев, Приближенный метод обратной задачи рассеяния в $J$-матричном подходе. Случай двух взаимодействующих каналов, ТМФ, 1999, том 121, номер 3, 424-435

DOI: https://doi.org/10.4213/tmf820

Использование Общероссийского математического портала Math-Net.Ru подразумевает, что вы прочитали и согласны с пользовательским соглашением

http: //www . mathnet.ru/rus/agreement

Параметры загрузки:

IP: 54.197 .130 .99

26 апреля 2023 г., 12:46:07 
ТЕОРЕТИЧЕСКАЯ

И МАТЕМАТИЧЕСКАЯ

ФИЗИКА

Том 121, № 3

декабрь, 1999

(C) 1999 г.

С. А. Зайцев*

\section{ПРИБЛИЖЕННЫЙ МЕТОД ОБРАТНОЙ ЗАДАЧИ РАССЕЯНИЯ В $J$-МАТРИЧНОМ ПОДХОДЕ. СЛУЧАЙ ДВУХ ВЗАИМОДЕЙСТВУЮШИХ КАНАЛОВ}

\footnotetext{
В рамках $J$-матричного подхода предложен методпостроения сепарабельных потенциалов с осцилляторными формфакторами по известной $S$-матрице. Рассмотрен случай упругого рассеяния с учетом двух взаимодействующих каналов.
}

\section{1. ВВЕДЕНИЕ}

В работе [1] был предложен способ построения в рамках $J$-матричного подхода [2-5] по известным фазе рассеяния и спектру квантовой системы сепарабельного потенциала

$$
\widehat{V}=\hbar \omega \sum_{n, n^{\prime}=0}^{N-1}\left|\varphi_{n}^{l}\right\rangle V_{n, n^{\prime}}\left\langle\varphi_{n^{\prime}}^{l}\right|
$$

с формфакторами $\left|\varphi_{n}^{l}\right\rangle$ в виде собственных функций гармонического осциллятора

$$
\left|\varphi_{n}^{l}\right\rangle=(-1)^{n} \sqrt{\frac{2 n !}{\rho \Gamma\left(n+l+\frac{3}{2}\right)}} \frac{x^{l+1}}{\exp \left(\frac{x^{2}}{2}\right)} L_{n}^{l+\frac{1}{2}}\left(x^{2}\right)
$$

где $x$ - относительная координата в единицах осцилляторного радиуса $\rho=\sqrt{\hbar / \mu \omega}, \mu$ приведенная масса.

Потенциалы (1) могут применяться в оболочечных расчетах. Действительно, центральным пунктом так называемой “по-core"-модели оболочек [6] является задача построения эффективного двухчастичного взаимодействия, которая решается с использованием реалистичных нуклон-нуклонных потенциалов [7]. Присутствие в результирующем выражении [6] проекторов на модельное пространство, ограниченное конечным числом осцилляторных базисных функций, означает, что это эффективное двухчастичное взаимодействие представимо в виде сепарабельного разложения (1).

* Хабаровский государственный технический университет, Хабаровск, Россия. E-mail: zaitsev@mail.khstu.ru 
В данной статье делается попытка распространить подход работы [1] на случай потенциалов

$$
\widehat{V}=\sum_{\alpha, \beta=1,2}|\alpha\rangle V^{\alpha \beta}\langle\beta|,
$$

где парциальные потенциалы $V^{\alpha \beta}$ задаются разложением

$$
V^{\alpha \beta}=\hbar \omega \sum_{n=0}^{N_{\alpha}-1} \sum_{n^{\prime}=0}^{N_{\beta}-1}\left|\varphi_{n}^{(\alpha)}\right\rangle V_{n, n^{\prime}}^{\alpha \beta}\left\langle\varphi_{n^{\prime}}^{(\beta)}\right|
$$

Символы $\alpha$ и $\beta$ обозначают наборы квантовых чисел, в которых присутствуют и значения орбитальных моментов $l_{\alpha}$ и $l_{\beta} ;\left|\varphi_{n}^{(\alpha)}\right\rangle \equiv\left|\varphi_{n}^{l_{\alpha}}\right\rangle$. Мы ограничиваемся "упругим" случаем, когда в обоих каналах энергия одинакова. Эту энергию будем измерять в единицах $\hbar \omega: \epsilon=E / \hbar \omega=q^{2} / 2$, где $q$ - сопряженный координате $x$ безразмерный импульс.

Ниже приведены используемые в дальнейшем $J$-матричные соотношения, отвечаюшие теории сильной связи каналов.

\section{2. ЭЛЕМЕНТЫ $J$-МАТРИЧНОГО ФОРМАЛИЗМА}

Компоненты $u_{\alpha \beta}(k, r)$ матрицы размерности $2 \times 2$ - решения уравнения Шредингера представляются в этом подходе в виде разложения

$$
u_{\alpha \beta}(k, r)=\sum_{n=0}^{\infty}\left|\varphi_{n}^{(\alpha)}\right\rangle b_{n}^{\alpha \beta}(q),
$$

коэффициенты $b_{n}^{\alpha \beta}$ которого удовлетворяют [8] соотношениям

$$
b_{n}^{\alpha \beta}(q)= \begin{cases}\sum_{\alpha^{\prime}} P_{n, N_{\alpha^{\prime}}-1}^{\alpha \alpha^{\prime}}(\epsilon) T^{\left(\alpha^{\prime}\right)} b_{N_{\alpha^{\prime}}}^{\alpha^{\prime} \beta}(q) & \text { при } n \leqslant N_{\alpha}-1, \\ \frac{i}{2}\left[C_{n}^{(\alpha)(-)}(q) \delta_{\alpha \beta}-C_{n}^{(\alpha)(+)}(q) S_{\alpha \beta}\right] & \text { при } n \geqslant N_{\alpha}-1,\end{cases}
$$

где $S_{\alpha \beta}$ - элементы дискретного аналога $S$-матрицы. В определении элементов

$$
P_{n, n^{\prime}}^{\alpha \beta}(\epsilon)=\left[(\mathbf{I} \epsilon-\mathbf{h})^{-1}\right]_{n, n^{\prime}}^{\alpha \beta},
$$

где $\mathbf{I}$ - единичная матрица, участвует матрица $\mathbf{h}$ гамильтониана $\widehat{H} / \hbar \omega$, рассчитанная в базисе $\left\{|\alpha\rangle\left|\varphi_{n}^{(\alpha)}\right\rangle, n=\overline{0, N_{\alpha}-1}, \alpha=\overline{1,2}\right\}$ [8]. Последняя представляет собой сумму $\mathbf{h}=\mathbf{T}+\mathbf{V}$, где $\mathbf{V}$ - матрица потенциала $\widehat{V} / \hbar \omega(4), \mathbf{T}$-матрица оператора кинетической энергии $\widehat{T} / \hbar \omega$, отличные от нуля элементы которой имеют вид

$$
\begin{aligned}
& T_{n, n-1}^{l_{\alpha}}=-\frac{1}{2} \sqrt{n\left(n+l_{\alpha}+\frac{1}{2}\right)}, \quad T_{n, n}^{l_{\alpha}}=\frac{1}{2}\left(2 n+l_{\alpha}+\frac{3}{2}\right), \\
& T_{n, n+1}^{l_{\alpha}}=-\frac{1}{2} \sqrt{(n+1)\left(n+l_{\alpha}+\frac{3}{2}\right)} .
\end{aligned}
$$

В выражениях (6) для элементов $T_{N_{\alpha}-1, N_{\alpha}}^{l_{\alpha}}$ используется обозначение $T^{(\alpha)}$. 
Функции

$$
C_{n}^{(\alpha)( \pm)}(q)=C_{n}^{l_{\alpha}}(q) \pm i S_{n}^{l_{\alpha}}(q)
$$

где

$$
\begin{aligned}
& C_{n}^{l}(q)=\sqrt{\frac{2 n !}{\Gamma\left(n+l+\frac{3}{2}\right)}} \frac{\Gamma\left(l+\frac{1}{2}\right)}{\pi q^{l} \exp \left(\frac{q^{2}}{2}\right)} F\left(-n-l-\frac{1}{2},-l+\frac{1}{2} ; q^{2}\right), \\
& S_{n}^{l}(q)=\sqrt{\frac{2 n !}{\Gamma\left(n+l+\frac{3}{2}\right)}} \frac{q^{l+1}}{\exp \left(\frac{q^{2}}{2}\right)} L_{n}^{l+\frac{1}{2}}\left(q^{2}\right),
\end{aligned}
$$

образуют фундаментальную систему решений дискретного аналога уравнения Шредингера [2]. При этом пара линейно независимых решений $C_{n}^{l}(q), S_{n}^{l}(q)$ удовлетворяет соотношению [9]

$$
C_{n+1}^{l}(q) S_{n}^{l}(q)-C_{n}^{l}(q) S_{n+1}^{l}(q)=\frac{q}{\pi T_{n, n+1}^{l}} .
$$

Выражения для элементов $S$-матрицы, которые получают из условия сшивки для коэффициентов $b_{n}^{\alpha \beta}$, имеют вид

$$
\begin{aligned}
S_{11}= & \frac{1}{\Delta(\epsilon)}\left\{\left(C_{N_{1}-1}^{(1)(-)}(q)-\mathcal{P}_{11}(\epsilon) T^{(1)} C_{N_{1}}^{(1)(-)}(q)\right) \times\right. \\
& \times\left(C_{N_{2}-1}^{(2)(+)}(q)-\mathcal{P}_{22}(\epsilon) T^{(2)} C_{N_{2}}^{(2)(+)}(q)\right)- \\
& \left.-\mathcal{P}_{12}^{2}(\epsilon) T^{(1)} T^{(2)} C_{N_{1}}^{(1)(-)}(q) C_{N_{2}}^{(2)(+)}(q)\right\}, \\
S_{22}= & \frac{1}{\Delta(\epsilon)}\left\{\left(C_{N_{1}-1}^{(1)(+)}(q)-\mathcal{P}_{11}(\epsilon) T^{(1)} C_{N_{1}}^{(1)(+)}(q)\right) \times\right. \\
& \times\left(C_{N_{2}-1}^{(2)(-)}(q)-\mathcal{P}_{22}(\epsilon) T^{(2)} C_{N_{2}}^{(2)(-)}(q)\right)- \\
& \left.-\mathcal{P}_{12}^{2}(\epsilon) T^{(1)} T^{(2)} C_{N_{1}}^{(1)(+)}(q) C_{N_{2}}^{(2)(-)}(q)\right\}, \\
S_{12}= & S_{21}=-\frac{2 i \sqrt{2 \epsilon} \mathcal{P}_{12}(\epsilon)}{\pi \Delta(\epsilon)},
\end{aligned}
$$

где

$$
\begin{aligned}
\Delta(\epsilon)= & \left(C_{N_{1}-1}^{(1)(+)}(q)-\mathcal{P}_{11}(\epsilon) T^{(1)} C_{N_{1}}^{(1)(+)}(q)\right) \times \\
& \times\left(C_{N_{2}-1}^{(2)(+)}(q)-\mathcal{P}_{22}(\epsilon) T^{(2)} C_{N_{2}}^{(2)(+)}(q)\right)- \\
& -\mathcal{P}_{12}^{2}(\epsilon) T^{(1)} T^{(2)} C_{N_{1}}^{(1)(+)}(q) C_{N_{2}}^{(2)(+)}(q) .
\end{aligned}
$$

Здесь $\mathcal{P}_{\alpha \beta}(\epsilon) \equiv P_{N_{1}-1, N_{2}-1}^{\alpha \beta}(\epsilon)$ - элементы так называемой $\mathcal{P}$-матрицы.

Если строится решение (5) с асимптотикой в форме стоячих волн, т.е. полагается, чтО

$$
b_{n}^{\alpha \beta}(q)=S_{n}^{l_{\alpha}}(q) \delta_{\alpha \beta}+C_{n}^{l_{\alpha}}(q) K_{\alpha \beta} \text { при } n \geqslant N_{\alpha}-1,
$$

где $K$-матрица связана с $S$-матрицей [10] соотношением

$$
\mathbf{S}=(\mathbf{I}+i \mathbf{K})(\mathbf{I}-i \mathbf{K})^{-1},
$$


то для элементов матрицы $\mathbf{K}$ получаются выражения

$$
\begin{aligned}
K_{11}= & -\frac{1}{\Delta_{1}(\epsilon)}\left\{\left(S_{N_{1}-1}^{l_{1}}(q)-\mathcal{P}_{11}(\epsilon) T^{(1)} S_{N_{1}}^{l_{1}}(q)\right) \times\right. \\
& \times\left(C_{N_{2}-1}^{l_{2}}(q)-\mathcal{P}_{22}(\epsilon) T^{(2)} C_{N_{2}}^{l_{2}}(q)\right)- \\
& \left.-\mathcal{P}_{12}^{2}(\epsilon) T^{(1)} T^{(2)} S_{N_{1}}^{l_{1}}(q) C_{N_{2}}^{l_{2}}(q)\right\}, \\
K_{22}= & -\frac{1}{\Delta_{1}(\epsilon)}\left\{\left(C_{N_{1}-1}^{l_{1}}(q)-\mathcal{P}_{11}(\epsilon) T^{(1)} C_{N_{1}}^{l_{1}}(q)\right) \times\right. \\
& \times\left(S_{N_{2}-1}^{l_{2}}(q)-\mathcal{P}_{22}(\epsilon) T^{(2)} S_{N_{2}}^{l_{2}}(q)\right)- \\
& \left.-\mathcal{P}_{12}^{2}(\epsilon) T^{(1)} T^{(2)} C_{N_{1}}^{l_{1}}(q) S_{N_{2}}^{l_{2}}(q)\right\}, \\
K_{12}= & K_{21}=-\frac{q \mathcal{P}_{12}(\epsilon)}{\pi \Delta_{1}(\epsilon)},
\end{aligned}
$$

где

$$
\begin{aligned}
\Delta_{1}(\epsilon)= & \left(C_{N_{1}-1}^{l_{1}}(q)-\mathcal{P}_{11}(\epsilon) T^{(1)} C_{N_{1}}^{l_{1}}(q)\right) \times \\
& \times\left(C_{N_{2}-1}^{l_{2}}(q)-\mathcal{P}_{22}(\epsilon) T^{(2)} C_{N_{2}}^{l_{2}}(q)\right)- \\
& -\mathcal{P}_{12}^{2}(\epsilon) T^{(1)} T^{(2)} C_{N_{1}}^{l_{1}}(q) C_{N_{2}}^{l_{2}}(q) .
\end{aligned}
$$

В дальнейшем символ $\mathbf{h}$ будет использоваться для обозначения $\mathcal{N} \times \mathcal{N}$-матрицы $(\mathcal{N}=$ $N_{1}+N_{2}$ ), элементы $h_{i j}$ которой определены в соответствии с правилом $h_{i j}=h_{n n^{\prime}}^{\alpha \beta}$, если

$$
i=n+1+\sum_{\alpha^{\prime}<\alpha} N_{\alpha^{\prime}} \text { и } j=n^{\prime}+1+\sum_{\alpha^{\prime}<\beta} N_{\alpha^{\prime}} .
$$

\section{3. ФАЗОВОЭКВИВАЛЕНТНЫЕ ПОТЕНЦИАЛЫ}

Из выражений (13)-(16) (или (19)-(22)) следует, что данные по рассеянию на потенциале (4) определяются элементами $\mathcal{P}_{\alpha \beta}(\epsilon)$, которые (если использовать спектральное разложение матрицы $\mathbf{h}=\mathbf{Z} \mathbf{\Lambda} \mathbf{Z}^{*}$, где символом $*$ обозначена операция транспонирования, $\mathbf{Z}$ - ортогональная матрица собственных векторов, $\boldsymbol{\Lambda}$ - диагональная матрица собственных значений $\left.\left\{\lambda_{j}\right\}\right)$ можно представить в виде

$$
\begin{aligned}
& \mathcal{P}_{11}(\epsilon)=\sum_{j=1}^{\mathcal{N}} \frac{Z_{N_{1}, j}^{2}}{\epsilon-\lambda_{j}} \\
& \mathcal{P}_{22}(\epsilon)=\sum_{j=1}^{\mathcal{N}} \frac{Z_{\mathcal{N}, j}^{2}}{\epsilon-\lambda_{j}}, \\
& \mathcal{P}_{12}(\epsilon)=\mathcal{P}_{21}(\epsilon)=\sum_{j=1}^{\mathcal{N}} \frac{Z_{N_{1}, j} Z_{\mathcal{N}, j}}{\epsilon-\lambda_{j}} .
\end{aligned}
$$

Заметим, что в функциях $\mathcal{P}_{\alpha \beta}(\epsilon)$ заключена информация, необходимая для построения (с точностью до некоторого ортогонального преобразования) матриц $\mathbf{h}$ и $\mathbf{V}$. Действительно, полюсы функций $\mathcal{P} \alpha \beta(\epsilon)$ совпадают с собственными значениями $\left\{\lambda_{j}\right\}$, а вычеты, 
в свою очередь, определяют $N_{1}$-ю и $\mathcal{N}$-ю строки (точнее, абсолютные значения и относительные знаки компонент $Z_{N_{1}, j}$ и $Z_{\mathcal{N}, j}$ ) матрицы $\mathbf{Z}$ собственных векторов.

Очевидно, что ортогональное преобразование с матрицей

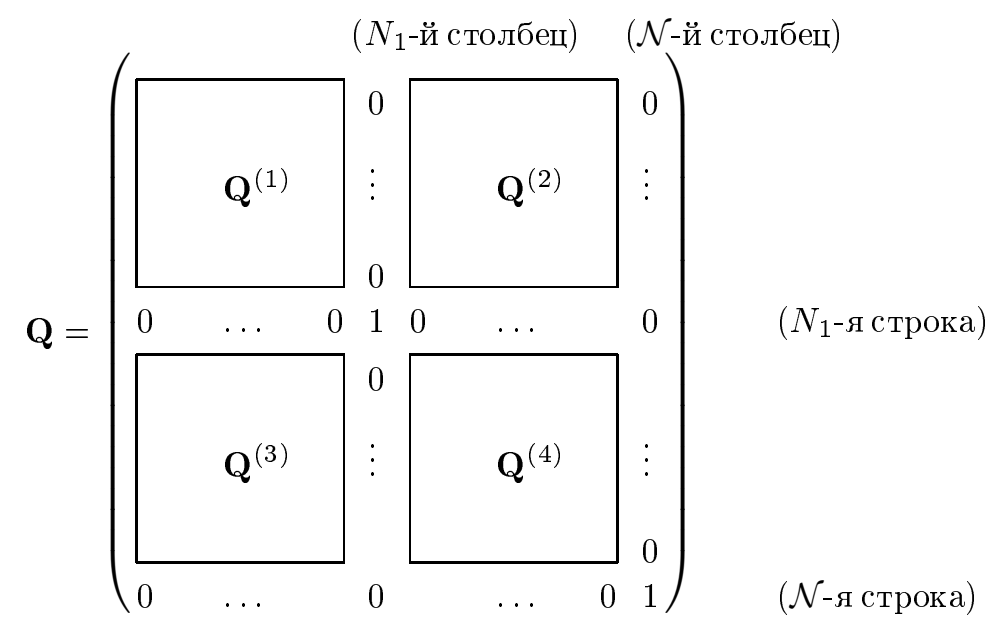

не затрагивает $N_{1}$-е и $\mathcal{N}$-е компоненты собственных векторов матришы hи, следовательно, оставляет неизменной $\mathcal{P}$-матрицу. Таким образом, данное преобразование связывает матрицы фазовоэквивалентных потенциалов $\mathbf{V}(Q)$, отвечающих фиксированным $N_{1}, N_{2}$ и $\hbar \omega:$

$$
\mathbf{V}(Q)=\mathbf{Q h}_{0} \mathbf{Q}^{*}-\mathbf{T}
$$

где $\mathbf{h}_{0}$ - фиксированная вешественная симметричная матрица размерности $\mathcal{N} \times \mathcal{N}$. Ортогональную матрицу $\mathbf{Q}$ можно представить в виде произведения матриц отражения $\mathbf{H}\left(u^{(k)}\right)$ [11], обрашающих векторы $\mathbf{u}^{(k)}$, компоненты $u_{N_{1}}^{(k)}, u_{\mathcal{N}}^{(k)}$ которых равны нулю.

По известным матрице $\boldsymbol{\Lambda}$ собственных значений и строкам $\left\{Z_{N_{1}, j}\right\},\left\{Z_{\mathcal{N}, j}\right\}$ матрищы собственных векторов матрица $\mathbf{h}_{0}=\mathbf{Z}_{0} \mathbf{\Lambda} \mathbf{Z}_{0}^{*}$ может быть получена с использованием матриц отражения следуюшим образом. Будем искать $\mathbf{Z}_{0}$ в виде произведения двух матриц отражения

$$
\mathbf{Z}_{0}=\mathbf{H}(v) \mathbf{H}(u) .
$$

При этом компоненты нормированного вектора $\mathbf{u}$ выражаются через элементы последней строки матрищы $\mathbf{Z}_{0}$ соотношениями

$$
u_{\mathcal{N}}^{2}=\frac{1-Z_{\mathcal{N}, \mathcal{N}}}{2}, \quad u_{i}=-\frac{Z_{\mathcal{N}, i}}{2 u_{\mathcal{N}}}, \quad i=\overline{1, \mathcal{N}-1}
$$

Нормированный вектор $\mathbf{v}$ строится по $N_{1}$-й строке матрицы $\mathbf{H}(v)$, элементы которой задаются выражениями

$$
H(v)_{N_{1}, i}=\sum_{j=1}^{\mathcal{N}} Z_{N_{1}, j} H(u)_{i, j} .
$$

Таким образом, для компонент вектора $\mathbf{v}$ имеем

$$
v_{N_{1}}^{2}=\frac{1-H(v)_{N_{1}, N_{1}}}{2}, \quad v_{\mathcal{N}}=0, \quad v_{i}=-\frac{H(v)_{N_{1}, i}}{2 v_{N_{1}}}, \quad i \neq N_{1}, \quad i \neq \mathcal{N} .
$$




\section{4. МЕТОД ПОСТРОЕНИЯ ПОТЕНЦИАЛОВ}

Пусть известна $S$-матриша $\mathbf{S}(E)$ при всех значениях $E>0$ и пусть заданы энергетические положения связанных состояний системы. Попытаемся построить связанные состояния и потеншиал (4), описываюший данные по рассеянию в некотором энергетическом интервале $\left[0, E_{0}\right]$ (за пределами которого матрица $\mathbf{S}(E)$ близка к единичной).

В полной аналогии с одноканальным случаем [1] зафиксируем некоторые значения параметров $\hbar \omega, N_{1}, N_{2}$ и определим на положительной полуоси энергий $\epsilon$ функции $\widetilde{\mathcal{P}}_{\alpha, \beta}$, обратив для этого выражения (19)-(21) относительно элементов $\mathcal{P}$-матрицы:

$$
\begin{aligned}
\widetilde{\mathcal{P}}_{11}(\epsilon)= & \frac{1}{T^{(1)} \Delta_{2}(\epsilon)}\left\{\left(S_{N_{1}-1}^{l_{1}}(q)+C_{N_{1}-1}^{l_{1}}(q) K_{11}\right)\left(S_{N_{2}}^{l_{2}}(q)+C_{N_{2}}^{l_{2}}(q) K_{22}\right)-\right. \\
& \left.-C_{N_{1}-1}^{l_{1}}(q) C_{N_{2}}^{l_{2}}(q) K_{12}^{2}\right\}, \\
\widetilde{\mathcal{P}}_{22}(\epsilon)= & \frac{1}{T^{(2)} \Delta_{2}(\epsilon)}\left\{\left(S_{N_{1}}^{l_{1}}(q)+C_{N_{1}}^{l_{1}}(q) K_{11}\right)\left(S_{N_{2}-1}^{l_{2}}(q)+C_{N_{2}-1}^{l_{2}}(q) K_{22}\right)-\right. \\
& \left.-C_{N_{1}}^{l_{1}}(q) C_{N_{2}-1}^{l_{2}}(q) K_{12}^{2}\right\}, \\
\widetilde{\mathcal{P}}_{12}(\epsilon)= & \widetilde{\mathcal{P}}_{21}(\epsilon)=-\frac{\sqrt{2 \epsilon} K_{12}}{\pi T^{(1)} T^{(2)} \Delta_{2}(\epsilon)},
\end{aligned}
$$

где

$$
\Delta_{2}(\epsilon)=\left(S_{N_{1}}^{l_{1}}(q)+C_{N_{1}}^{l_{1}}(q) K_{11}\right)\left(S_{N_{2}}^{l_{2}}(q)+C_{N_{2}}^{l_{2}}(q) K_{22}\right)-C_{N_{1}}^{l_{1}}(q) C_{N_{2}}^{l_{2}}(q) K_{12}^{2} .
$$

Как и в потенциальном рассеянии [1], проблема сводится к построению рациональных функций $\mathcal{P}_{\alpha \beta}(\epsilon)(23)-(25)$, аппроксимируюших функции $\widetilde{\mathcal{P}}_{\alpha \beta}(\epsilon)(32)-(34)$ на интервале $\left[0, \epsilon_{0}\right]$. Свойства вычетов последних мы рассмотрим подробнее. Полюсы матрицы $\widetilde{\mathcal{P}}$ совпадают с нулями $\left\{\tilde{\lambda}_{j}\right\}$ знаменателя $\Delta_{2}(\epsilon)(35)$, т.е. при $\epsilon=\tilde{\lambda}_{j}$ справедливо равенство

$$
\begin{aligned}
& \left(S_{N_{1}}^{l_{1}}\left(\sqrt{2 \tilde{\lambda}_{j}}\right)+C_{N_{1}}^{l_{1}}\left(\sqrt{2 \tilde{\lambda}_{j}}\right) K_{11}\left(\tilde{\lambda}_{j}\right)\right)\left(S_{N_{2}}^{l_{2}}\left(\sqrt{2 \tilde{\lambda}_{j}}\right)+C_{N_{2}}^{l_{2}}\left(\sqrt{2 \tilde{\lambda}_{j}}\right) K_{22}\left(\tilde{\lambda}_{j}\right)\right)= \\
& \quad=C_{N_{1}}^{l_{1}}\left(\sqrt{2 \tilde{\lambda}_{j}}\right) C_{N_{2}}^{l_{2}}\left(\sqrt{2 \tilde{\lambda}_{j}}\right) K_{12}^{2}\left(\tilde{\lambda}_{j}\right) .
\end{aligned}
$$

С учетом (36) получаем для вычетов

$$
\begin{aligned}
\mathcal{R}_{j}^{(1)} & =\operatorname{Res}_{\epsilon=\tilde{\lambda}_{j}} \widetilde{\mathcal{P}}_{11}(\epsilon)= \\
& =\frac{\sqrt{2 \tilde{\lambda}_{j}}\left(S_{N_{2}}^{l_{2}}\left(\sqrt{2 \tilde{\lambda}_{j}}\right)+C_{N_{2}}^{l_{2}}\left(\sqrt{2 \tilde{\lambda}_{j}}\right) K_{22}\left(\tilde{\lambda}_{j}\right)\right)}{\pi\left(T^{(1)}\right)^{2} C_{N_{1}}^{l_{1}}\left(\sqrt{2 \tilde{\lambda}_{j}}\right)} \operatorname{Res}_{\epsilon=\tilde{\lambda}_{j}} \frac{1}{\Delta_{2}(\epsilon)}, \\
\mathcal{R}_{j}^{(2)} & =\operatorname{Res}_{\epsilon=\tilde{\lambda}_{j}} \widetilde{\mathcal{P}}_{22}(\epsilon)= \\
& =\frac{\sqrt{2 \tilde{\lambda}_{j}}\left(S_{N_{1}}^{l_{1}}\left(\sqrt{2 \tilde{\lambda}_{j}}\right)+C_{N_{1}}^{l_{1}}\left(\sqrt{2 \tilde{\lambda}_{j}}\right) K_{11}\left(\tilde{\lambda}_{j}\right)\right)}{\pi\left(T^{(2)}\right)^{2} C_{N_{2}}^{l_{2}}\left(\sqrt{2 \tilde{\lambda}_{j}}\right)} \operatorname{Res}_{\epsilon=\tilde{\lambda}_{j}} \frac{1}{\Delta_{2}(\epsilon)}, \\
\mathcal{R}_{j}^{(12)} & =\operatorname{Res}_{\epsilon=\tilde{\lambda}_{j}} \widetilde{\mathcal{P}}_{12}(\epsilon)=-\frac{\sqrt{2 \tilde{\lambda}_{j}} K_{12}\left(\tilde{\lambda}_{j}\right)}{\pi T^{(1)} T^{(2)}} \operatorname{Res}_{\epsilon=\tilde{\lambda}_{j}} \frac{1}{\Delta_{2}(\epsilon)} .
\end{aligned}
$$


Из уравнения (36) для полюсов матрицы $\widetilde{\mathcal{P}}$ и выражений (37)-(39) следует соотношение, связьваюшее вычеты,

$$
\mathcal{R}_{j}^{(1)} \mathcal{R}_{j}^{(2)}=\left(\mathcal{R}_{j}^{(12)}\right)^{2} .
$$

Требование положительности величин $\left\{\mathcal{R}_{j}^{(1)}\right\}$ и $\left\{\mathcal{R}_{j}^{(2)}\right\}$, необходимое для возможного отождествления последних соответственно с квадратами компонент $\left\{Z_{1, j}\right\}$ и $\left\{Z_{\mathcal{N}, j}\right\}$, удовлетворяется при разделении корней числителя корнями знаменателя у функций $\widetilde{\mathcal{P}}_{11}(\epsilon)$ и $\widetilde{\mathcal{P}}_{22}(\epsilon)$. Последнее, как легко показать с помошью асимптотических выражений для $C_{n}^{l}(q)$ и $S_{n}^{l}(q)$ при $n \rightarrow \infty[9]$, имеет место при достаточно больших $N_{1}, N_{2}$.

Необходимым условием совпадения функций $\widetilde{\mathcal{P}}_{\alpha, \beta}(\epsilon)$ и $\mathcal{P}_{\alpha, \beta}(\epsilon)$ на интервале $\left[0, \epsilon_{0}\right]$ является совпадение на данном интервале полюсов и вычетов этих функций. Подробнее остановимся на свойствах полюсов матришы $\widetilde{\mathcal{P}}$. При этом нас будет интересовать поведение последних в случае появления в системе связанных состояний. Рассмотрим с этой целью случай потенциального $s$-волнового рассеяния на потенциале (4) ранга $N$, когда матрица $\widetilde{\mathcal{P}}$ сводится к единственной функции [1]

$$
\widetilde{\mathcal{P}}_{N}(\epsilon)=\frac{1}{T_{N-1, N}^{0}} \frac{\widetilde{C}_{N-1,0}(\epsilon)}{\widetilde{C}_{N, 0}(\epsilon)}
$$

где

$$
\widetilde{C}_{n, l}(\epsilon)=\cos \delta_{l}(\epsilon) S_{n}^{l}(q)+\sin \delta_{l}(\epsilon) C_{n}^{l}(q) .
$$

При отсутствии связанных состояний корням $\left\{\widetilde{\lambda}_{n}>0\right\}$ знаменателя $\widetilde{C}_{N, 0}(\epsilon)$ можно поставить в соответствие корни $\left\{\lambda_{n}^{(0)}, n=\overline{1, N}\right\}$ синусоподобного решения $S_{N}^{0}(q)\left(q_{n}^{(0)}=\right.$ $\left.\sqrt{2 \lambda_{n}^{(0)}}\right)$. Последние совпадают с корнями полинома Лагерра $L_{N}^{1 / 2}(2 \epsilon)$, входящего в определение (11) функции $S_{N}^{0}(q)$. Переход к большим значениям $N$ позволяет воспользоваться следуюшими более наглядными асимптотическими выражениями [9]:

$$
\begin{aligned}
S_{N}^{0}(q) & \approx \frac{2}{\sqrt{\pi}(4 N+3)^{1 / 4}} \sin (\sqrt{2(4 N+3) \epsilon}) \\
\widetilde{C}_{N, 0}(\epsilon) & \approx \frac{2}{\sqrt{\pi}(4 N+3)^{1 / 4}} \sin \left(f_{N}(\epsilon)\right)
\end{aligned}
$$

и

$$
f_{N}(\epsilon)=\sqrt{2(4 N+3) \epsilon}+\delta(\epsilon),
$$

из которых следует, что достаточным условием отмеченного соответствия корней, очевидно, является требование $f_{N}^{\prime}(\epsilon)>0$ на интервале $\left[0, \epsilon_{0}\right]$, поскольку $\left\{\lambda_{n}^{(0)}\right\}$ и $\left\{\widetilde{\lambda}_{n}\right\}$ удовлетворяют асимптотическим уравнениям

$$
\begin{aligned}
\sqrt{2(4 N+3) \lambda_{n}^{(0)}}=n \pi, \quad n=\overline{1, N}, \\
f_{N}\left(\widetilde{\lambda}_{n}\right)=n \pi, \quad n=1,2, \ldots,
\end{aligned}
$$




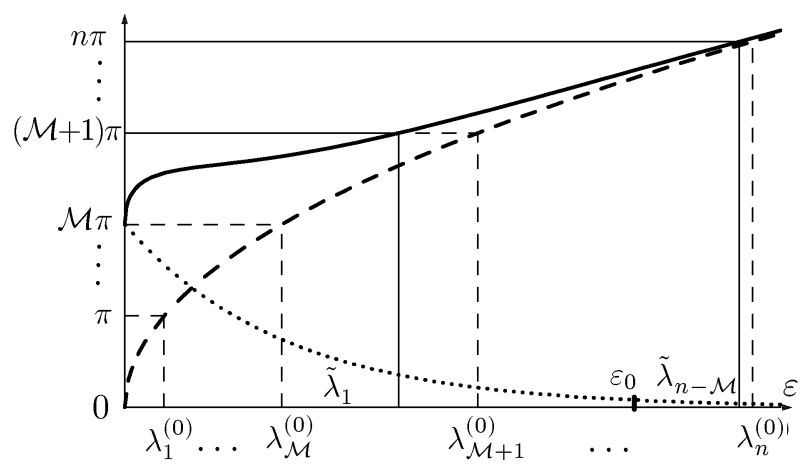

Рис. 1

и $f_{N}(0)=\delta(0)=0$ (т. к. связанные состояния отсутствуют). Заметим, что справедливость неравенств $f_{N-1}^{\prime}(\epsilon)>0, f_{N}^{\prime}(\epsilon)>0$, как было показано в [1], является достаточным условием разделения корней числителя корнями знаменателя выражения (41) (или положительности вычетов функции $\widetilde{\mathcal{P}}_{N}(\epsilon)$ ).

Пусть в системе присутствует $\mathcal{M} \geqslant 1$ связанных состояний $\left\{\varepsilon_{i}<0, i=\overline{1, \mathcal{M}}\right\}$, тогда $\delta(0)-\delta(\infty)=\mathcal{M} \pi$. На рис. 1 схематически изображено графическое решение уравнений (45) и (46). Здесь левая часть $f_{N}(\epsilon)$ уравнения $(46)$, фаза $\delta(\epsilon)$ и левая часть уравнения (45) изображены соответственно сплошной, пунктирной и штриховой линиями. Из уравнений $(45),(46)$ и условия $\delta(0)=\mathcal{M} \pi$ следует, что при $f_{N}^{\prime}(\epsilon)>0$ у функции $\widetilde{C}_{N, 0}(\epsilon)$ "исчезает" $\mathcal{M}$ корней, которые можно было бы сопоставить с первыми $\mathcal{M}$ значениями $\left\{\lambda_{i}^{(0)}, i=\overline{1, \mathcal{M}}\right\}$ (см. рис. 1$)$.

Энергии $\left\{\varepsilon_{j}\right\}$ связанных состояний удовлетворяют [1] уравнениям

$$
\mathcal{P}_{N}\left(\varepsilon_{j}\right)=\frac{1}{T_{N-1, N}^{0}} \frac{C_{N-1}^{0(+)}\left(i \sqrt{-2 \varepsilon_{j}}\right)}{C_{N}^{0(+)}\left(i \sqrt{-2 \varepsilon_{j}}\right)},
$$

которые при больших $N[9]$ принимают вид

$$
\mathcal{P}_{N}\left(\varepsilon_{j}\right)=\frac{1}{T_{N-1, N}^{0}} \exp \left(\sqrt{\frac{-2 \varepsilon_{j}}{N}}\right) .
$$

Левая часть уравнения (48) есть [1] по определению

$$
\mathcal{P}_{N}(\epsilon)=\sum_{j=1}^{N} \frac{Z_{N, j}^{2}}{\epsilon-\lambda_{j}}
$$

и при всех $\lambda_{j}>0$ в области $\epsilon<0$ ведет себя как $\epsilon^{-1}$. Правая часть (48) здесь также отрицательна и возрастает по абсолютной величине. Очевидно, что в случае $\lambda_{j}>0$, $j=\overline{1, N}$, сушествует единственный корень уравнения (47), если

$$
\mathcal{P}_{N}(0)<\frac{1}{T_{N-1, N}^{0}} \frac{C_{N-1}^{0(+)}(0)}{C_{N}^{0(+)}(0)}=-\frac{2}{N} .
$$


$\delta_{1,0}$, град.

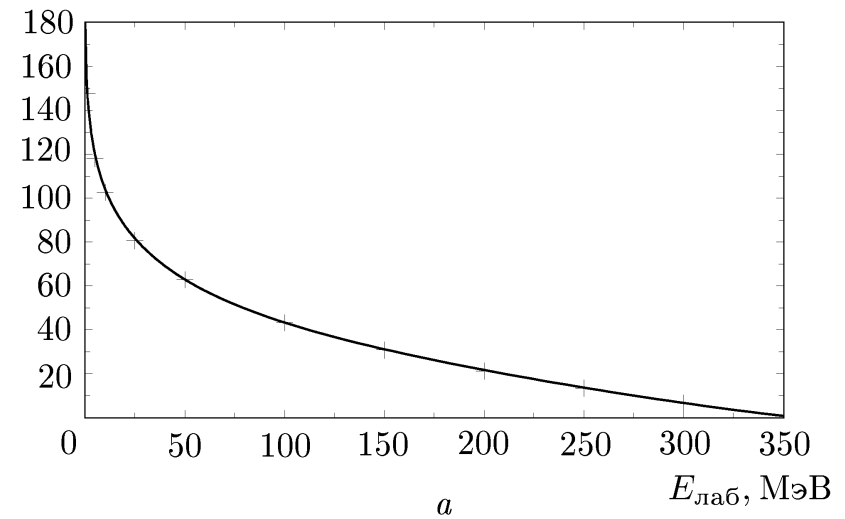

$\delta_{1,2}$, град.

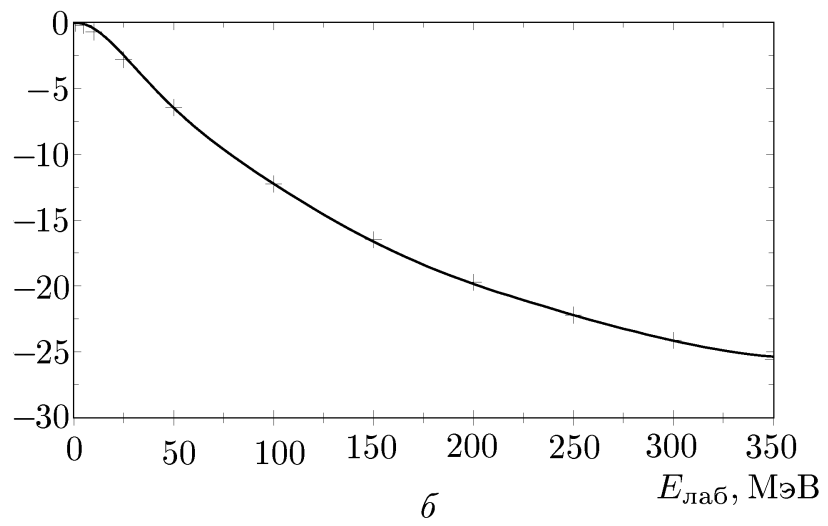

$\varepsilon_{1}$, град.

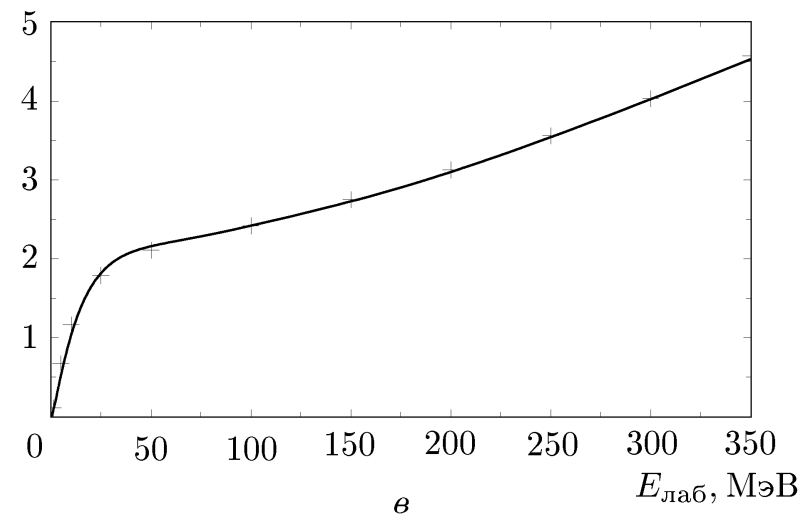

Рис. 2

Таким образом, с положительными $\left\{\lambda_{j}\right\}$ можно описать не более одного связанного состояния. Если число $\mathcal{M}$ связанных состояний превышает единицу, то для описания каждого состояния $\varepsilon_{i}$ (кроме, может быть, первого) в $\mathcal{P}_{N}(\epsilon)$ необходимо присутствие слагаемого $Z_{N, i}^{2} /\left(\epsilon-\lambda_{i}\right)$ с отрицательным параметром $\lambda_{i}\left(\varepsilon_{i}<\lambda_{i}<0\right)$. Эта необходимость 
в отрицательных параметрах у функции $\mathcal{P}_{N}(\epsilon)$ (дробно-рационального образа функции $\left.\widetilde{\mathcal{P}}_{N}(\epsilon)\right)$ согласуется с “исчезновением" полюсов у $\widetilde{\mathcal{P}}_{N}(\epsilon)$ на интервале $\left[0, \epsilon_{0}\right]$.

При отсутствии связанных состояний имеет место соответствие между корнями свободных решений $S_{N_{1}}^{l_{1}}, S_{N_{2}}^{l_{2}}$ и полюсами матрицы $\widetilde{\mathcal{P}}$; причем в области $\epsilon>\epsilon_{0}$ полюсы и соответствуюшие им корни практически совпадают, поскольку здесь $\mathbf{S} \approx \mathbf{I}$. Пусть за пределами интервала $\left[0, \epsilon_{0}\right]$ лежат $\mathcal{K}$ полюсов $\tilde{\lambda}_{n}$. Тогда (подобно случаю потенциального рассеяния [1]) можно фиксировать “внутренние" $(j \leqslant \mathcal{N}-\mathcal{K})$ параметры, положив $\lambda_{j}=\tilde{\lambda}_{j}$ и $Z_{N_{1}, j}=\sqrt{\mathcal{R}_{j}^{(1)}}, Z_{\mathcal{N}, j}=\operatorname{sign}\left(\mathcal{R}_{j}^{(12)}\right) \sqrt{\mathcal{R}_{j}^{(2)}}$ при $j=\overline{1, \mathcal{N}-\mathcal{K}}$. Ориентиром для определения "внешних" $(n>\mathcal{N}-\mathcal{K})$ параметров $\mathcal{P}$-матришы будут служить полюсы $\left\{\widetilde{\lambda}_{n}, n=\overline{\mathcal{N}-\mathcal{K}+1, \mathcal{N}}\right\}$ и выгеты $\left\{\mathcal{R}_{n}^{(1)}, \mathcal{R}_{n}^{(12)}, \mathcal{R}_{n}^{(2)}, \quad n=\overline{\mathcal{N}-\mathcal{K}+1, \mathcal{N}}\right\}$ матрицы $\widetilde{\mathcal{P}}$. При этом число $\mathcal{K}$ должно быть достаточно большим $\left(\mathcal{K}_{\min }=2\right)$, чтобы удовлетворялись условия ортонормированности строк матрицы $\mathbf{Z}$ собственных векторов:

$$
\sum_{j=1}^{\mathcal{N}} Z_{N_{1}, j}^{2}=\sum_{j=1}^{\mathcal{N}} Z_{\mathcal{N}, j}^{2}=1, \quad \sum_{j=1}^{\mathcal{N}} Z_{N_{1}, j} Z_{\mathcal{N}, j}=0
$$

Величины $\hbar \omega, N_{1}$ и $N_{2}$ являются параметрами задачи. Причем, если задаться целью минимизировать ранг $\mathcal{N}$ потенциала $(4)$, то велиина $\hbar \omega$ остается практически единственным вариационным параметром, оптимальное значение $\hbar \omega_{0}$ которого позволяет получить наилучшее (для заданных $N_{1}$ и $N_{2}$ ) описание данных по рассеянию. Более тонкая "настройка" осушествляется выбором "внешних" параметров $\left\{\lambda_{n}\right\}$. В случае $\hbar \omega=\hbar \omega_{0}$ вариации $\delta \lambda_{n}$ являются малыми по сравнению с соответствуюшими начальными значениями $\widetilde{\lambda}_{n}$.

Если в системе присутствуют связанные состояния $\varepsilon_{j}<0, j=\overline{1, \mathcal{M}}$, то "недостаток" полюсов у матрицы $\widetilde{\mathcal{P}}$ внутри интервала $\left[0, \epsilon_{0}\right]$ следует восполнить отрицательными параметрами $\left\{\lambda_{j}\right\}$. Дополнительными условиями здесь являются $\mathcal{M}$ уравнений на связанные состояния [9], имеющие вид

$$
\begin{aligned}
\Delta\left(\varepsilon_{j}\right)= & \left(C_{N_{1}-1}^{(1)(+)}\left(i \sqrt{-2 \varepsilon_{j}}\right)-\mathcal{P}_{11}\left(\varepsilon_{j}\right) T^{(1)} C_{N_{1}}^{(1)(+)}\left(i \sqrt{-2 \varepsilon_{j}}\right)\right) \times \\
& \times\left(C_{N_{2}-1}^{(2)(+)}\left(i \sqrt{-2 \varepsilon_{j}}\right)-\mathcal{P}_{22}\left(\varepsilon_{j}\right) T^{(2)} C_{N_{2}}^{(2)(+)}\left(i \sqrt{-2 \varepsilon_{j}}\right)\right)- \\
& -\mathcal{P}_{12}^{2}\left(\varepsilon_{j}\right) T^{(1)} T^{(2)} C_{N_{1}}^{(1)(+)}\left(i \sqrt{-2 \varepsilon_{j}}\right) C_{N_{2}}^{(2)(+)}\left(i \sqrt{-2 \varepsilon_{j}}\right)=0 .
\end{aligned}
$$

Обращает на себя внимание аналогия предлагаемого метода и способа получения исходной спектральной информации $\left\{E_{\lambda}, \gamma_{\lambda}\right\}$ ( $E_{\lambda}$ - энергетические положения, $\gamma_{\lambda}$ - приведенные ширины $R$-резонансов) для восстановления потенциала $v(n)$ в рамках конечно-разностного аналога $R$-матричной теории рассеяния [12].

\section{5. ПРИМЕР}

Выше использовался метод построения потенциала (4), описывающего взаимодействие в $n p$-системе, когда последняя находится в триплетном спиновом состоянии и обладает полным моментом $J=1$. Отличные от нуля недиагональные по индексу канала элементы $V_{n n^{\prime}}^{\alpha \neq \beta}$ матрицы потенциала в этом случае имитируют тензорное взаимодействие, связываюшее парциальные амплитуды с орбитальными моментами $l_{1}=0$ и $l_{2}=2$. 
Значения $\bar{\delta}_{1,0}, \bar{\delta}_{1,2}$ фаз рассеяния и параметра смешивания $\bar{\varepsilon}_{1}$ (в "ядерной" параметризации [13]) при $E_{\text {лаб }} \leqslant 350$ МэВ взяты из работы [7]. При $E_{\text {лаб }}>350$ МэВ величины $\bar{\delta}_{1,0}, \bar{\delta}_{1,2}, \bar{\varepsilon}_{1}$ экстраполировались функциями с затухающей асимптотикой. Результаты расчета, отвечающие потенциалу (4) с параметрами, представленными в таблице, изображены на рис. 2 сплошной линией; крестиками обозначены данные работы [7].

В таблице приведены значения полюсов $\left\{\widetilde{\lambda}_{j}\right\}$, вычетов $\left\{\mathcal{R}_{j}^{(1)}\right\},\left\{\mathcal{R}_{j}^{(2)}\right\}$ матришы $\widetilde{\mathcal{P}}$, параметров $\left\{\lambda_{j}\right\},\left\{Z_{N_{1}, j}^{2}\right\},\left\{Z_{\mathcal{N}, j}^{2}\right\}$ и знаков произведений $\left\{s_{j}=\operatorname{sign}\left(Z_{N_{1}, j} Z_{\mathcal{N}, j}\right)\right\}$, входяших в выражения (23)-(25) для элементов $\mathcal{P}$-матрицы, отвечающей потенциалу $(4)$ с $\hbar \omega=62 \mathrm{MэB}, l_{1}=0, l_{2}=2, N_{1}=N_{2}=4$.

\begin{tabular}{|c|l|l|l|l|l|l|l|}
\hline$j$ & \multicolumn{1}{|c|}{$\widetilde{\lambda}_{j}$} & \multicolumn{1}{c|}{$\lambda_{j}$} & \multicolumn{1}{c|}{$\mathcal{R}_{j}^{(1)}$} & \multicolumn{1}{c|}{$Z_{N_{1}, j}^{2}$} & \multicolumn{1}{c|}{$\mathcal{R}_{j}^{(2)}$} & \multicolumn{1}{c|}{$Z_{\mathcal{N}, j}^{2}$} & $s_{j}$ \\
\hline & 0.00320263 & & 0.000388487 & & 0.000396550 & & \\
& 0.00414204 & & -0.00322305 & & -0.000203556 & & \\
1 & 0.0125593 & 0.00719953 & 0.00988730 & 0.00875 & 0.000115510 & 0.0000536257 & + \\
2 & 0.799503 & 0.799503 & 0.0186411 & 0.0186411 & 0.0543272 & 0.0543272 & - \\
3 & 0.838921 & 0.838921 & 0.0953768 & 0.0953768 & 0.0119322 & 0.0119322 & + \\
4 & 2.10551 & 2.10551 & 0.00280480 & 0.00280480 & 0.167453 & 0.167453 & - \\
5 & 2.51864 & 2.51864 & 0.329940 & 0.329940 & 0.00278319 & 0.00278319 & + \\
6 & 4.04111 & 4.0265 & 0.00397203 & 0.00397203 & 0.290857 & 0.290857 & - \\
7 & 5.18711 & 5.25 & 0.550008 & 0.539765 & 0.00432657 & 0.00319449 & + \\
8 & 6.81030 & 6.85 & 0.00443192 & 0.00075 & 0.480994 & 0.469400 & - \\
\hline
\end{tabular}

Как уже отмечалось, при достаточно больших значениях $N_{1}, N_{2}$ (и отсутствии связанных состояний в системе) имеет место соответствие между полюсами матрищы $\widetilde{\mathcal{P}}$ и корнями произведения $S_{N_{1}}^{l_{1}}, S_{N_{2}}^{l_{2}}$ (в которое вырождается функция $\Delta_{2}(\epsilon)(35)$ при выключении взаимодействия). Причем достаточное условие существования такого соответствия, полученное для случая потенциального рассеяния [1], в рассматриваемом примере может быть записано в виде неравенств

$$
\begin{aligned}
& \frac{f_{N_{\alpha}}(\epsilon)}{d \epsilon}>\frac{f_{N_{\alpha}-1}(\epsilon)}{d \epsilon}>0, \\
& f_{N_{\alpha}}(\epsilon)=\sqrt{2\left(4 N_{\alpha}+2 l_{\alpha}+3\right) \epsilon}+\delta_{\alpha} \epsilon-\frac{\pi l_{\alpha}}{2}, \quad \alpha=1,2,
\end{aligned}
$$

поскольку связь между каналами здесь довольно слаба, что отражается в малости параметра смешивания $\bar{\varepsilon}_{1}$ (см. рис. 2). Нашей целью было построение потенциала (4), задаваемого минимальным числом параметров (т. е. матрицей минимального ранга $\mathcal{N}$ ). Легко убедиться в том, что для $N_{1}=4$ и $\hbar \omega=62$ МэВ производная $f_{N_{1}}^{\prime}(\epsilon)$ отрицательна в окрестности точки $\epsilon=0$, где фаза $\bar{\delta}_{1,0}(\epsilon)$ убывает особенно быстро. В результате соответствие между полюсами матрицы $\widetilde{\mathcal{P}}$ и корнями свободных решений $S_{N_{1}}^{l_{1}}, S_{N_{2}}^{l_{2}}$ оказывается нарушенным, что выражается в появлении у $\widetilde{\mathcal{P}}$ двух “лишних" полюсов в окрестности начала координат оси энергий. Эти “лишние" полюсы и соответствующие им вычеты (представленные в первых двух строках таблицы) мы исключили из рассмотрения.

О том, насколько значение $\hbar \omega=62$ МэВ близко к "оптимальному" для данных $N_{1}=$ $N_{2}=4$, можно судить по малости различия "внешних" (лежаших правее интервала 
$\left.\left[0, \epsilon_{0}=2.82452\right]\right)$ параметров $\left\{\lambda_{6}, \lambda_{7}, \lambda_{8}\right\} \quad \mathcal{P}$-матрицы и соответствуюших полюсов $\left\{\widetilde{\lambda}_{n}, n \geqslant 6\right\}$ матрицы $\widetilde{\mathcal{P}}$.

Значение искомого параметра $\lambda_{1}$ (положительное) получено с использованием уравнения (50) на связанное состояние с энергией $\varepsilon=-2.22458 / \hbar \omega=-0.03588025$. Поведение фаз рассеяния и особенно параметра смешивания в окрестности начала координат оси энергий оказалось довольно чувствительным к выбору значений параметров $Z_{N_{1}, 1}$ и $Z_{\mathcal{N}, 1}$.

\section{6. ЗАКЛЮЧЕНИЕ}

Для устранения сушествуюшей в нашем подходе неоднозначности в определении матрицы потенциала (4) необходима дополнительная исходная информация. В случае нуклон-нуклонной системы в качестве такой можно было бы использовать волновую функцию связанного состояния дейтрона (полученную, например, из численного расчета с реалистичным нуклон-нуклонным потенциалом [7]). В обшем случае $\mathcal{N}-2$ "внутренних" коэффициентов [9] $\left(N_{1}-1\right.$ для $s$-состояния и $N_{2}-1$ для $d$-состояния) разложения волновой функции по базисным осцилляторным функциям (2) недостаточно для однозначного определения элементов искомой матришы $\mathbf{Q}(26)$ фазовоэквивалентного преобразования, например, если $\mathbf{Q}$ не является матрицей отражений, задаваемой $\mathcal{N}-2$ отличными от нуля компонентами обрашаемого ею вектора [11]. Таким образом, и в этом случае сохраняется возможность строить нуклон-нуклонные потенциалы (4) с различающимися внемассовыми свойствами.

Благодарности. Автор выражает благодарность А.И. Мазуру и А. М. Широкову за полезные обсуждения и помошь в работе.

\section{Список литературы}

[1] С. А. Зайцев. ТМФ. 1998. Т. 115. № 2. С. 263.

[2] H. A. Yamani, L. Fishman. J. Math. Phys. 1975. V. 16. № 2. P. 410.

[3] Г. Ф. Филиппов. ЯФ. 1980. Т. 32. С. 932; 1981. Т. 33. С. 928.

[4] Ю.И. Нечаев, Ю. Ф. Смирнов. ЯФ. 1982. Т. 35. С. 1385.

[5] J. Revai, M. Sotona, J. Zofka. J. Phys. G. 1985. V. 11. № 4. P. 745.

[6] P. Navratil, B. R. Barrett. Phys. Rev. C. 1996. V. 54. № 6. P. 2986.

[7] V. G. J. Stoks, R. A. M. Klomp, C.P.F.Terheggen, J. J. de Swart. Phys. Rev. C. 1994. V. 49. P. 2950.

[8] J. T. Broad, W. P. Reinhardt. J. Phys. B. 1976. V. 9. № 9. P. 1491

[9] С. А.Зайчев, Ю. Ф. Смирнов, А. М. Широков. ТМФ. 1998. Т. 117. № 2. С. 227.

[10] P. Ньютон. Теория рассеяния волн и частиц. М.: Мир, 1966.

[11] Б. Парлетm. Симметричная проблема собственных значений. Численные методы. М.: Мир, 1983.

[12] Б. Н. Захарьев, А. А. Сузько. Потенциалы и квантовое рассеяние. Прямая и обратная задачи. М.: Энергоатомиздат, 1985.

[13] В. В. Бабиков. Метод фазовых функций в квантовой механике. М.: Наука, 1976.

Поступила в редакцию 18.III.1999 г. 